LAWRENCE LIVERMORE N A TIO N A L LABORATORY
Cylinder Test on C-4

P. C. Souers

May 10, 2007 
This document was prepared as an account of work sponsored by an agency of the United States Government. Neither the United States Government nor the University of California nor any of their employees, makes any warranty, express or implied, or assumes any legal liability or responsibility for the accuracy, completeness, or usefulness of any information, apparatus, product, or process disclosed, or represents that its use would not infringe privately owned rights. Reference herein to any specific commercial product, process, or service by trade name, trademark, manufacturer, or otherwise, does not necessarily constitute or imply its endorsement, recommendation, or favoring by the United States Government or the University of California. The views and opinions of authors expressed herein do not necessarily state or reflect those of the United States Government or the University of California, and shall not be used for advertising or product endorsement purposes.

This work was performed under the auspices of the U.S. Department of Energy by University of California, Lawrence Livermore National Laboratory under Contract W-7405-Eng-48. 


\title{
Cylinder Test on C-4
}

\author{
C.Souers May 3, 2007 \\ Prepared for Marlin Kipp \\ Sandia National Laboratories \\ Albuquerque, NM \\ (505) 844-9005
}

This is an old shot using a streak camera. The details are:

\begin{tabular}{|c|c|c|c|c|c|c|c|c|}
\hline $\begin{array}{l}\text { Explosive } \\
\text { Name }\end{array}$ & $\begin{array}{c}\text { Cylinder } \\
\text { Shot } \\
\text { No. }\end{array}$ & $\begin{array}{l}\text { Expl. } \\
\text { Density } \\
\text { (g/cc) }\end{array}$ & $\begin{array}{l}\text { Cylinder } \\
\text { Material }\end{array}$ & $\begin{array}{c}\text { Approx } \\
\text { Diam } \\
\text { inches }\end{array}$ & $\begin{array}{c}\text { Detvel } \\
\text { (mm/us) }\end{array}$ & $\begin{array}{c}\text { Inner } \\
\text { Diameter } \\
(\mathrm{mm})\end{array}$ & $\begin{array}{c}\text { Wall } \\
\text { Thickness } \\
(\mathrm{mm})\end{array}$ & $\begin{array}{l}\text { Shot } \\
\text { Date }\end{array}$ \\
\hline COMP C-4 & 289 & 1.601 & $\mathrm{Cu}$ & 1.00 & 8.193 & 25.430 & 2.593 & 740314 \\
\hline time (us) & \multicolumn{2}{|c|}{ velocity (mm/us) } & ) time (us) & \multicolumn{3}{|c|}{$\mathrm{R}-\mathrm{Ro}(\mathrm{mm})$} & & \\
\hline & 0.426 & 0.721 & & 0.000 & & 0.000 & & \\
\hline & 1.052 & 0.897 & & 0.852 & & 0.614 & & \\
\hline & 1.301 & 1.014 & & 1.252 & & 0.973 & & \\
\hline & 1.533 & 1.088 & & 1.350 & & 1.073 & & \\
\hline & 1.761 & 1.063 & & 1.717 & & 1.472 & & \\
\hline & 2.005 & 1.092 & & 1.805 & & 1.565 & & \\
\hline & 2.423 & 1.123 & & 2.204 & & 2.002 & & \\
\hline & 2.821 & 1.180 & & 2.642 & & 2.493 & & \\
\hline & 3.040 & 1.191 & & 2.999 & & 2.915 & & \\
\hline & 3.218 & 1.220 & & 3.080 & & 3.011 & & \\
\hline & 3.431 & 1.258 & & 3.356 & & 3.348 & & \\
\hline & 3.643 & 1.285 & & 3.506 & & 3.537 & & \\
\hline & 3.883 & 1.280 & & 3.779 & & 3.887 & & \\
\hline & 4.081 & 1.306 & & 3.987 & & 4.153 & & \\
\hline & 4.273 & 1.320 & & 4.175 & & 4.398 & & \\
\hline & 4.467 & 1.318 & & 4.372 & & 4.659 & & \\
\hline & 4.673 & 1.341 & & 4.561 & & 4.908 & & \\
\hline & 4.855 & 1.340 & & 4.786 & & 5.209 & & \\
\hline & 5.077 & 1.365 & & 4.924 & & 5.395 & & \\
\hline & 5.418 & 1.359 & & 5.230 & & 5.812 & & \\
\hline & 5.779 & 1.393 & & 5.607 & & 6.324 & & \\
\hline & 6.025 & 1.496 & & 5.951 & & 6.803 & & \\
\hline & 6.209 & 1.475 & & 6.099 & & 7.026 & & \\
\hline & 6.429 & 1.436 & & 6.319 & & 7.350 & & \\
\hline & 6.658 & 1.425 & & 6.538 & & 7.664 & & \\
\hline
\end{tabular}




\begin{tabular}{|c|c|c|c|}
\hline 6.879 & 1.385 & 6.778 & 8.007 \\
\hline 7.082 & 1.405 & 6.979 & 8.285 \\
\hline 7.328 & 1.457 & 7.184 & 8.573 \\
\hline 7.537 & 1.484 & 7.473 & 8.993 \\
\hline 7.781 & 1.479 & 7.601 & 9.184 \\
\hline 8.203 & 1.498 & 7.960 & 9.715 \\
\hline 8.657 & 1.475 & 8.445 & 10.442 \\
\hline 8.936 & 1.469 & 8.869 & 11.066 \\
\hline 9.128 & 1.529 & 9.003 & 11.264 \\
\hline 9.333 & 1.539 & 9.252 & 11.644 \\
\hline 9.513 & 1.466 & 9.414 & 11.894 \\
\hline 9.749 & 1.512 & 9.611 & 12.183 \\
\hline 9.930 & 1.596 & 9.887 & 12.600 \\
\hline 10.138 & 1.557 & 9.972 & 12.736 \\
\hline 10.532 & 1.517 & 10.303 & 13.251 \\
\hline 10.969 & 1.573 & 10.762 & 13.947 \\
\hline 11.391 & 1.542 & 11.175 & 14.597 \\
\hline 11.816 & 1.548 & 11.607 & 15.264 \\
\hline 12.103 & 1.488 & 12.024 & 15.909 \\
\hline 12.293 & 1.545 & 12.182 & 16.143 \\
\hline 12.548 & 1.612 & 12.404 & 16.488 \\
\hline 12.725 & 1.650 & 12.691 & 16.949 \\
\hline 12.972 & 1.645 & 12.760 & 17.064 \\
\hline 13.384 & 1.480 & 13.184 & 17.761 \\
\hline 13.773 & 1.642 & 13.584 & 18.353 \\
\hline 14.016 & 1.569 & 13.961 & 18.971 \\
\hline 14.192 & 1.497 & 14.071 & 19.144 \\
\hline 14.379 & 1.564 & 14.313 & 19.507 \\
\hline 14.551 & 1.651 & 14.446 & 19.714 \\
\hline 14.800 & 1.599 & 14.656 & 20.062 \\
\hline 14.978 & 1.518 & 14.943 & 20.521 \\
\hline 15.188 & 1.603 & 15.012 & 20.625 \\
\hline 15.562 & 1.598 & 15.364 & 21.189 \\
\hline 15.965 & 1.623 & 15.760 & 21.823 \\
\hline 16.224 & 1.586 & 16.169 & 22.485 \\
\hline 16.419 & 1.573 & 16.280 & 22.662 \\
\hline 16.630 & 1.605 & 16.557 & 23.098 \\
\hline 16.812 & 1.617 & 16.704 & 23.333 \\
\hline 17.045 & 1.589 & 16.920 & 23.682 \\
\hline 17.226 & 1.616 & 17.171 & 24.081 \\
\hline 17.474 & 1.587 & 17.281 & 24.259 \\
\hline
\end{tabular}




\begin{tabular}{ll|ll}
17.886 & 1.628 & 17.668 & 24.873 \\
18.294 & 1.623 & 18.104 & 25.583 \\
18.658 & 1.631 & 18.484 & 26.199 \\
18.877 & 1.650 & 18.832 & 26.768 \\
19.101 & 1.608 & 18.922 & 26.915 \\
19.487 & 1.658 & 19.280 & 27.492 \\
19.900 & 1.606 & 19.694 & 28.177 \\
20.333 & 1.629 & 20.107 & 28.842 \\
20.759 & 1.671 & 20.558 & 29.577 \\
21.162 & 1.589 & 20.960 & 30.249 \\
21.594 & 1.662 & 21.364 & 30.890 \\
22.021 & 1.626 & 21.823 & 31.654 \\
22.400 & 1.618 & 22.219 & 32.298 \\
22.627 & 1.754 & 22.580 & 32.882 \\
22.806 & 1.685 & 22.674 & 33.045 \\
23.040 & 1.584 & 22.938 & 33.490 \\
23.169 & 1.594 & 23.143 & 33.815 \\
23.304 & 1.738 & 23.195 & 33.898 \\
23.528 & 1.631 & 23.414 & 34.279 \\
23.760 & 1.639 & 23.643 & 34.653 \\
23.946 & 1.686 & 23.877 & 35.036 \\
24.073 & 1.594 & 24.015 & 35.269 \\
24.202 & 1.496 & 24.130 & 35.453 \\
24.344 & 1.709 & 24.273 & 35.666 \\
& & 24.414 & 35.907
\end{tabular}

